\title{
The Karonga Prevention Trial - which BCG?
}

\author{
J M PÖNNIGHAUS \& P E M FINE \\ Lepra Evaluation Project, Chilumba, Karonga District, Malawi, and \\ Ross Institute, London School of Hygiene and Tropical Medicine, Lon- \\ don, UK
}

A leprosy vaccine trial, the Karonga Prevention Trial, was started in Karonga District in Northern Malawi in January 1986. The objectives are essentially:

1. to test whether $\mathrm{BCG}+\operatorname{killed} M$. leprae gives greater protection against leprosy than $\mathrm{BCG}$ alone and

2. to test whether a repeat $\mathrm{BCG}$ vaccination can add to the protection against clinical leprosy given by the first BCG vaccination.

The protective efficacy of BCG alone against clinical leprosy in this population had been found to be at least $50 \%$ [1]. For this reason a 'no vaccination/ placebo' group has not been included in the trial. The $50 \%$ protective efficacy was achieved with Glaxo BCG given during mass campaigns in the mid-seventies and within the $\mathrm{MCH}$ services since then. This was and still is a strong argument in favour of also using Glaxo BCG for the Karonga Prevention Trial, though there is no published evidence to suggest that one type of BCG imparts more protection against leprosy than another [2].

It was observed however, that ulcer sizes in Venezuela after vaccination with Statens Serum Institute Copenhagen BCG, were much smaller than those observed after vaccination with Glaxo BCG during preliminary studies in Malawi [3, 4]. Because of this observation it was decided to carry out comparative studies between these two different BCG vaccines in Malawi. The results of these studies, undertaken in late 1985 and in 1986, are the subject of this presentation.

\section{Methods}

The vaccinations were carried out within the context of the Lepra Evaluation Project, a total population survey in Karonga District [5]. Current standard Copenhagen BCG vaccine (batch 616) and current standard Glaxo BCG vaccine (batch D698) were given. Allocation of BCG was on the basis of two Glaxo BCG ten dosage vials followed by one Copenhagen BCG 20 dosage vial, followed by two Glaxo BCG vials etc. Allocation of BCG was not blind because Glaxo BCG is bottled in clear glass 10 dosage vials while Copenhagen BCG is bottled in brown glass 20 dosage vials. Standard dosages were administered in $0.1 \mathrm{ml}$ intradermally in the right deltoid region by trained paramedical workers (Leprosy Control Assistants, LCAs) under the close supervision of JMP. The vaccines were given after an examination for clinical leprosy and any individuals with a history or clinical signs of leprosy were excluded from the study. Also excluded were children under 3 months of age, any children with obvious clinical malnutrition and any individuals seriously ill. 

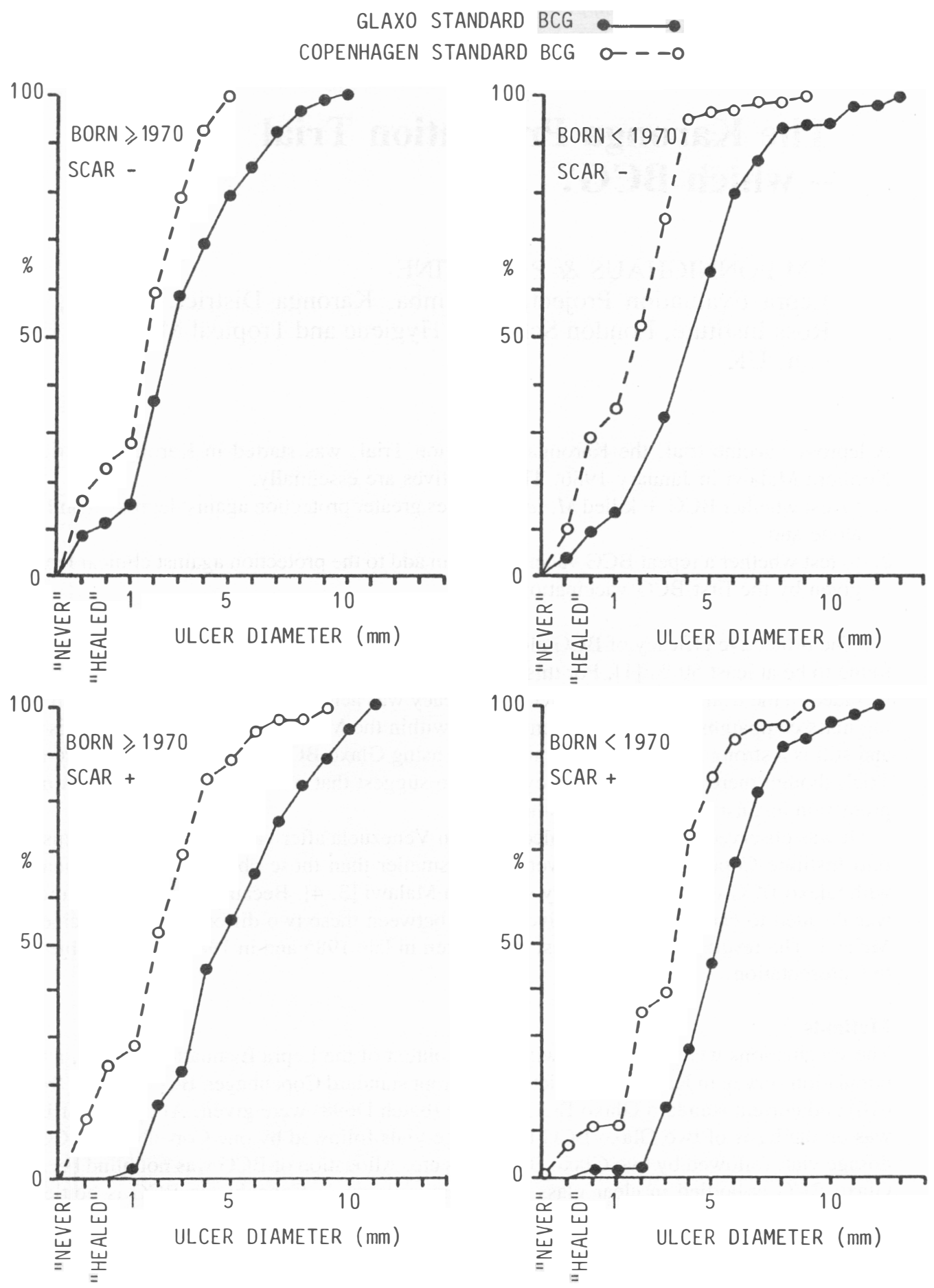

Figure 1. Cumulative relative frequencies of ulcer sizes four weeks after vaccination with current standard doses of Glaxo and current standard doses of Statens Serum Institute Copenhagen BCG, as observed in Karonga District, Nothern Malawi, 1985. BCG was injected in $0.1 \mathrm{ml}$ intradermally in the right deltoid region. 
$M$. leprae soluble antigen (MLSA) skin tests were carried out at the time of vaccination with BCG (day 0) and at three and nine months later. Two MLSA preparations were used: «WEL-1», prepared according to Dr Convit's protocol and «CD 19» prepared by Dr Rees [4]. On day $\mathrm{O}$ one or the other preparation was used, and after 3 and 9 months both preparations were given together. Administration was by intradermal injection into the forearms. MLSA skin test induration sizes were read by the LCAs after 48 or 72 hours. Both diameters of any induration were measured and the mean was used for the analysis.

Vaccine ulcers (and indurations around the ulcers, not shown in this presentation) were measured after 4 weeks. Care was taken to remove scabs so that the true (remaining) ulcer size could be measured. If at 4 weeks new skin had already covered the ulcer it was called «healed» and no attempt was made to measure the size of the previous ulcer. Vaccine ulcer scar sizes were measured nine months after vaccination.

The reading of ulcer sizes after four weeks and all later vaccination scar and $M$. leprae soluble antigen skin test reading was carried out blind of the type of BCG which had been given.

BCG vaccine and MLSA preparations were kept cold in the field by keeping vials and syringes in insulated flasks with ice blocks inside.

\section{Results}

The vaccine ulcer sizes are presented in Figure 1 as cumulative relative frequency distributions. For all groups, whether born before or after 1970 and whether or not they had a prior BCG scar, the Copenhagen BCG gave rise to smaller ulcers than did the Glaxo BCG. Thus in terms of unpleasantness of vaccination the Copenhagen BCG had a clear advantage over the Glaxo preparation in this population.

MLSA skin test results are presented as «conversion rates» at 3 and 9 months after vaccination. Conversion rates are defined as proportions of those «negative» (less than or equal to $5 \mathrm{~mm}$ ) on day 0 who were "positive» (greater than $5 \mathrm{~mm}$ induration) on day 90 or on day 270 respectively. Table 1 shows that on day 90 coversion rates in most subgroups following vaccination with Glaxo BCG were higher than after vaccination with Copenhagen BCG. However, although suggestive of greater sensitization by Glaxo BCG, the overall difference in conversion rates is not statistically significant.

Table 2 shows conversion rates 9 months after vaccination. Most of the individuals tested after nine months were different from those tested after three months. Conversion rates associated with the two vaccines had fallen to the same level ( $15 \%$ and $14 \%$ respectively) as assessed with the WEL-1 antigen. In terms of CD19 the conversion rates had fallen to $36 \%$ and $15 \%$ after vaccination with the Glaxo and Copenhagen BCG respectively. This difference is not statistically significant (chi-square $=2.48$ ). However the results are again in the direction of greater sensitization by the Glaxo than by the Copenhagen BCG.

For those retested with CD19 it is also possible to look at the «reversion» rates, the proportion of those «positive» on day 0 who were «negative» on day 270 . Table 3 shows that the reversion rates were $32 \%$ in spite of vaccination with Glaxo BCG and $39 \%$ in spite of vaccination with Copenhagen BCG nine months after vaccination. Only 8 out of 138 individuals were positive on day 0 when tested with WEL-1, of whom two (25\%) were negative after nine months. 
Table 1. Conversion rates to M. leprae soluble antigens (MLSA) subsequent to vaccination with current standard Glaxo or current standard Copenhagen BCG, as observed in Karonga District, Northern Malawi, 1985-86. M. leprae antigens used were CD19 (Rees type) and WEL-I (Convit type). Conversion rates defined as proportions of those "negative» to MLSA at time of vaccination (denominators) who were «positive» 90 days thereafter (numerators). Skin test positivity defined as greater than $5 \mathrm{~mm}$ induration. Data broken down by age (year of birth) and prior BCG scar status.

\begin{tabular}{lcccccc}
\hline \multirow{2}{*}{ MLSA } & \multirow{2}{*}{ BCG } & \multicolumn{2}{c}{ Born $\geqslant 1970$} & \multicolumn{2}{c}{ Born $<1970$} & \multirow{2}{*}{ Total } \\
& & Scar - & Scar + & Scar - & Scar + & \\
\hline \multirow{4}{*}{ CD19 } & Glaxo & $15 / 19$ & $8 / 11$ & $10 / 13$ & $3 / 3$ & $36 / 46$ \\
& & $(0.79)$ & $(0.73)$ & $(0.77)$ & $(1.0)$ & $(0.78)$ \\
& & & & & & \\
& Copen- & $4 / 7$ & $10 / 14$ & $8 / 15$ & $1 / 2$ & $23 / 38$ \\
& hagen & $(0.57)$ & $(0.71)$ & $(0.53)$ & $(0.5)$ & $(0.61)$ \\
\hline \multirow{3}{*}{ WEL-1 } & Glaxo & $10 / 21$ & $3 / 22$ & $5 / 36$ & $3 / 5$ & $21 / 84$ \\
& & $(0.48)$ & $(0.14)$ & $(0.14)$ & $(0.6)$ & $(0.25)$ \\
& & & & & & \\
& Copen- & $2 / 15$ & $4 / 25$ & $5 / 20$ & $2 / 4$ & $13 / 64$ \\
& hagen & $(0.13)$ & $(0.16)$ & $(0.25)$ & $(0.50)$ & $(0.20)$ \\
\hline
\end{tabular}

Table 2. Conversion rates to $\mathrm{M}$. leprae soluble antigens (MLSA) subsequent to vaccination with current standard Glaxo or current standard Copenhagen BCG, as observed in Karonga District, Northern Malawi, 1985-86. M. leprae antigens used were CD19 (Rees type) and WEL-1 (Convit type). Conversion rates defined as proportions of those «negative» to MLSA at time of vaccination (denominators) who were "positive» 270 days thereafter (numerators). Skin test positivity defined as greater than $5 \mathrm{~mm}$ induration. Data broken down by age (year of birth) and prior BCG scar status.

\begin{tabular}{lcccccr}
\hline \multirow{2}{*}{ MLSA } & \multirow{2}{*}{ BCG } & \multicolumn{2}{c}{ Born $\geqslant 1970$} & \multicolumn{2}{c}{ Born $<1970$} & \multirow{2}{*}{ Sotal } \\
& & Scar - & Scar + & Scar - & Scar + & \\
\hline \multirow{4}{*}{ CD19 } & $4 / 9$ & $3 / 8$ & $1 / 4$ & $0 / 1$ & $8 / 22$ \\
& & $(0.44)$ & $(0.37)$ & $(0.25)$ & & $(0.36)$ \\
& Copen- & $1 / 4$ & $1 / 7$ & $1 / 7$ & $0 / 2$ & $3 / 20$ \\
& hagen & $(0.25)$ & $(0.14)$ & $(0.14)$ & & $(0.15)$ \\
\hline \multirow{3}{*}{ WEL-1 } & Glaxo & $1 / 34$ & $2 / 14$ & $6 / 19$ & $2 / 7$ & $11 / 74$ \\
& & $(0.03)$ & $(0.14)$ & $(0.31)$ & $(0.28)$ & $(0.15)$ \\
& Copen- & $1 / 21$ & $3 / 16$ & $4 / 16$ & $0 / 4$ & $8 / 57$ \\
& hagen & $(0.05)$ & $(0.19)$ & $(0.25)$ & & $(0.14)$ \\
\hline
\end{tabular}


Table 3. Reversion rates to M. leprae soluble antigens (MLSA) subsequent to vaccination with current standard Glaxo or current standard Copenhagen BCG, as observed in Karonga District, Northern Malawi, 1985-86. M. leprae antigens used was CD19 (Rees type). Reversion rates defined as proportions of those «positive» to MLSA at time of vaccination (denominators) who were "negative» 270 days thereafter (numerators). Skin test positivity defined as greater than $5 \mathrm{~mm}$ induration. Data broken down by age (year of birth) and prior BCG scar status.

\begin{tabular}{lcccccc}
\hline \multirow{2}{*}{ MILSA } & \multirow{2}{*}{ BCG } & \multicolumn{2}{c}{ Born $\geqslant 1970$} & \multicolumn{2}{c}{ Born $<1970$} & \multirow{2}{*}{ Total } \\
& Scar - & Scar + & Scar - & Scar + & \\
\hline \multirow{3}{*}{ CD19 } & $1 / 2$ & $3 / 9$ & $5 / 13$ & $2 / 10$ & $11 / 34$ \\
& & $(0.50)$ & $(0.33)$ & $(0.38)$ & $(0.20)$ & $(0.32)$ \\
& $\begin{array}{c}\text { Copen- } \\
\text { hagen }\end{array}$ & $1 / 2$ & $3 / 5$ & $3 / 10$ & $2 / 6$ & $9 / 23$ \\
& $(0.50)$ & $(0.60)$ & $(0.30)$ & $(0.33)$ & $(0.39)$ \\
\hline
\end{tabular}

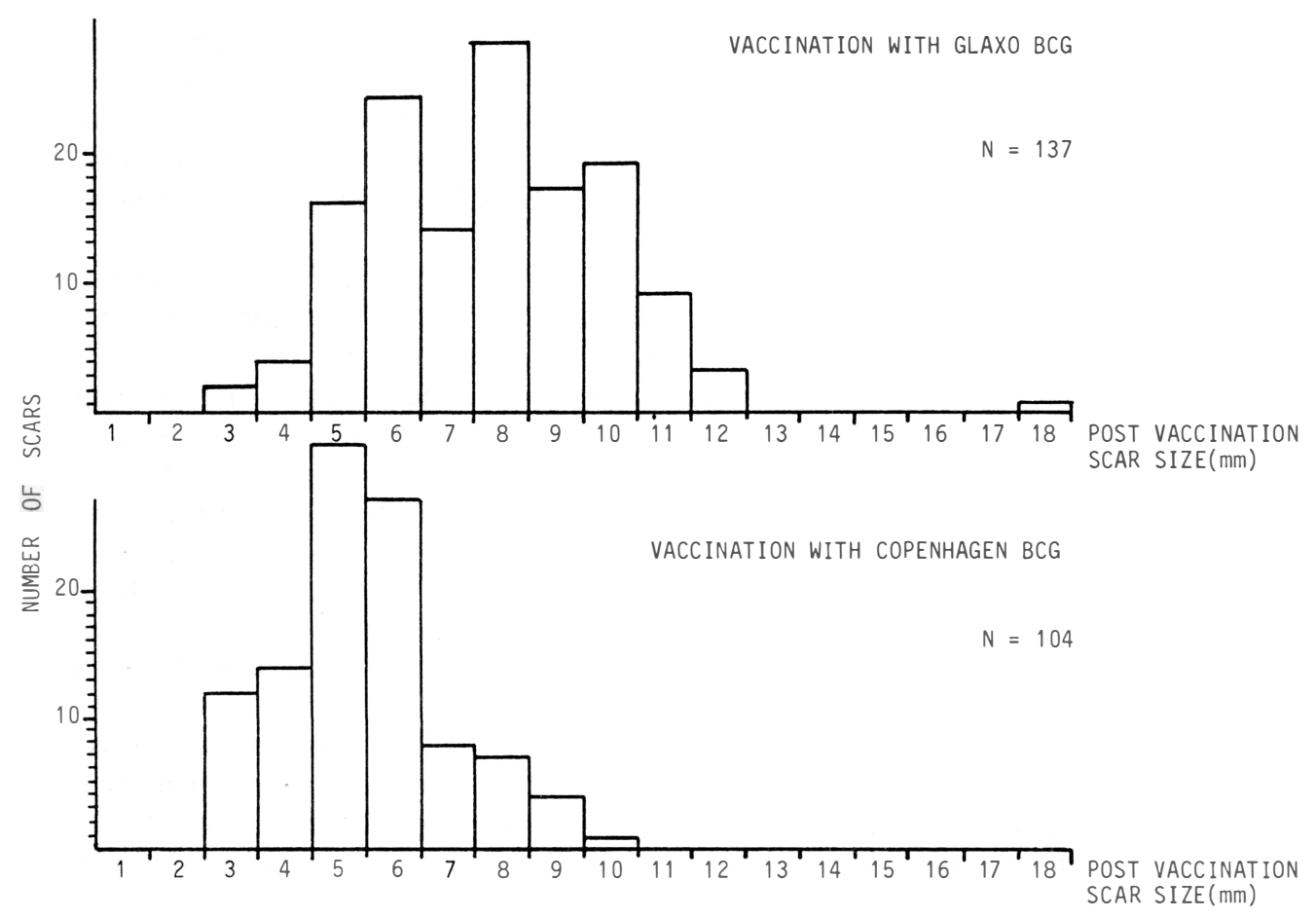

Figure 2. Frequency distribution of vaccination scar sizes (average diameters) nine months after vaccination with current standard doses of Glaxo and current standard doses of Statens Serum Institute Copenhagen BCG as observed in Karonga District, Northern Malawi 1986. $B C G$ was injected in $0.1 \mathrm{ml}$ intradermally in the right deltoid region. 
The frequency distributions of scars at vaccination sites are shown in Figure 2. In those individuals with more than one scar, in other words in individuals already with a BCG scar prior to (re-) vaccination with Glaxo or Copenhagen BCG, it was not always obvious which of the two or even three scars was the most recent one, although usually a decision could be made on the basis of some remaining hypopigmentation of the scar. The arithmetic mean of scars nine months after vaccination was $7.77 \mathrm{~mm}$ for the Glaxo preparation and $5.45 \mathrm{~mm}$ for the Copenhagen BCG. This difference is statistically highly significant $(\mathrm{p}<0.0001)$ and confirms the greater ulcerogenicity of the Glaxo compared with the Copenhagen BCG in this population.

\section{Discussion}

As reading of ulcer sizes, post vaccination MLSA skin test induration sizes and post vaccination scar sizes was done blind and by the same staff, any observed differences in ulcer sizes, in conversion rates and in post vaccination scar sizes between Glaxo BCG and Copenhagen BCG cannot be explained by observer bias or inter-observer variation.

Vaccination ulcer sizes increase with age and are larger in individuals with than in those without a scar from a previous BCG vaccination. In each subgroup the Glaxo BCG caused larger ulers than the Copenhagen BCG. This difference in ulcer sizes associated with the two vaccines could be due to differences between the BCG strains themselves or to methods of culturing, harvesting and preparing the bacilli. Published studies comparing different BCG strains have shown that one factor which determines ulcer size is the number or proportion of dead bacilli [6]. We are not aware of direct comparisons between total and viable counts of the two vaccines used in the study, and recognize that this is itself a complicated problem, because of differences in the culture methods employed. When vaccines from these sources were compared in the mid-Sixties a Copenhagen BCG gave larger ulcers than did a Glaxo preparation [7]. However, Glaxo has more than doubled the moist weight content of its standard preparation since these earlier studies [8].

In any case, the most important question seems to be whether ulcer sizes are relevant to immunity or are mere «side effects». Could there be an association - or even a causal relationship - between ulcer sizes and protective immunity against clinical leprosy?

The higher conversion rates found three months after vaccination with Glaxo in comparison with Copenhagen BCG are seen nine months after vaccination when re-testing with CD19 but not with WEL-1. A greater sensitisation by Glaxo than by Copenhagen BCG is also suggested by smaller reversion rates nine months after vaccination, though none of these differences is statistically significant. In addition, earlier studies suggested to us that the specificity of WEL-1 is higher than the specificity of CD19 [4]. Thus, even it the difference between the Glaxo and Copenhagen BCG's, as assessed by skin testing with CD19, were genuine it might not signify a higher sensitization by the Glaxo BCG against $M$. leprae antigens.

Altogether the conversion rates had fallen to very low levels nine months after vaccination with both BCG preparations. In fact they have fallen to much lower levels than the ones observed in Venezuela where $77 \%$ of individuals vaccinated with Copenhagen BCG $(0.5$ or $2.7 \mathrm{x}$ the current standard dose depending on the initial tuberculin status) had skin test induration sizes $\geqslant 10 \mathrm{~mm}$ one year af ter vaccination [9]. However, a different MLSA preparation was used in these Venezuelan studies and their results are thus not directly comparable with ours.

In contrast with our conversion rates the protective immunity observed after vaccination with Glaxo BCG in the Malawi population is long term and at least $50 \%$. This confirms again that delayed type hypersensitivity reactions and protective immunity are largely if not entire- 
ly unrelated phenomena [10] and that conversion rates are of poor predicitive value for the amount of protective immunity likely to be observed. Since we have no data on the protective immunity of Copenhagen BCG in this population we can however not be sure whether post vaccination ulcer sizes are of similarly poor predictive value.

For practical purpose it could be bery important to know whether there are advantages in using preparations which give larger ulcers. At least in our experience, ulcers have a strong negative influence on the acceptability of a vaccine by the people. If there were advantages, that is if there was a strong association between ulcer sizes and protective immunity developing against clinical leprosy then ulcers would have to be made acceptable by a strong component of health education within any vaccination programme. However if there were no advantages, if ulcers were a mere side effect, then BCG preparations which give smaller ulcers would be much preferable for large scale use, given that once a leprosy vaccine was administered within the framework of General Health Services people would have to come forward actively for vaccination.

Meanwhile given that our only data on BCG's protection in this population relate to the Glaxo preparation, it seems appropiate to continue using Glaxo BCG in this population for the leprosy vaccine trial, in spite of the size of the ulcers it may cause.

\section{Acknowledgments}

The authours wish to thank all participantants of the study for their cooperation and Messrs D. Chavula and P. Mwafulirwa for their special efforts while undertaking the three and nine months post vaccination skin testing. Basic funding for the Lepra Evaluation Project and the Karonga Prevention. Trial is provided by the British Leprosy Relief Association and other ILEP members. The Karonga Prevention Trial is also supported by a grant from the Immunology of Leprosy (IMMLEP) component of the UNDP/World Bank/WHO Programme for Research and Training in Tropical Diseases. Dr RJW Rees provided the M. leprae soluble antigen preparations and Judith Russell helped to prepare the manuscript.

\section{References}

1 Fine PEM, Pönnighaus JM, Maine N, Clarkson JA, and Bliss L. The protective efficacy of BCG against leprosy in Northern Malawi. Lancet 1986, 2, 499-502.

2 Fine PEM. The Kellersberger Memorial Lecture, 1985. The role of BCG in the Control of Leprosy. Ethiop Med J 1985, 23, 179-191.

3 Pönnighaus J M. Visit to Venezuelan Vaccine Trial. Report to The Chief Medical Officer, Leprosy Division of Communicable Diseases, WHO. (Ref: T16/370/L4/i).

4 Pönnighaus JM and Fine PEM. Sensitization studies with potential leprosy vaccine preparations in Northern Malawi. Int $\mathbf{J}$ Lepr 1986, 54, 25-37.

5 Pönnighaus J M, Fine P E M, Bliss L, Sliney IJ, Bradley DJ, Rees RJW. The Lepra Evaluation Project - I Methods (in preparation).

6 Edwards LB, Palmer CE and Magnus K. BCG Vaccination Studies by the WHO Tuberculosis Research Office, Copenhagen. World Health Organization Monograph Series (1953), 129-141.

7 Horwitz $\mathrm{O}$ and Bunch-Christensen $\mathrm{K}$. Correlation between tuberculin sensitivity after 2 months and 5 years among BCG vaccinated subjects. Bull Wld Hlth Org 1972, 47, 49-58.

8 Wright D. Glaxo Operations UK Limited. Letter to Dr Paul Fine (1st August 1986).

9 Smith PG «Report of a visit to review progress on the leprosy immunoprophylaxis trial in Venezuela (Report 5)» prepared by PG Smith and quoted with permission from Dr Convit. 
10 Fine PEM. Pönnighaus JM, Maine N. The relationship between delayed type hypersensitivity and protective immunity induced by mycobacterial vaccines in man. Lepr Rev (1986) 57, Suppl 2. 